\title{
The Sound Power Radiated by Some Axisymmetric Vibration Modes of a Guided Annular Plate
}

\author{
Wojciech P. Rdzanek Jr. \\ Department of Acoustics, University of Rzeszów, Al. Rejtana 16A, 35-310 Rzeszów, Poland
}

(Received 7 October 2002; accepted 22 November 2002)

\begin{abstract}
This paper presents an asymptotic approach to find the values of the active and reactive sound power of a fully guided annular plate. The results obtained are valid for some time-harmonic axisymmetric high-frequency processes. The plate is embedded in a planar rigid baffle. Low fluid loading and low internal friction are assumed. The self power of the plate has been presented in its elementary form together with its approximation error. Some sample numerical results obtained from the integral and asymptotic formulae show good agreement within the whole high frequency range. The elementary formulae are highly efficient, and therefore they can be used for computing the total sound power radiated by the plate.
\end{abstract}

\section{INTRODUCTION}

The problems of measuring and identifying the sound fields generated by some vibrating flat circular annular plates, which satisfy some different axisymmetric or asymmetric boundary conditions and which are characterised by different excitation behaviours, are still of considerable interest both from theoretical and practical points of view. Therefore, some approximate methods to deal with the sound radiation by some selected plates have seldom been presented in the literature. The equivalent surface area method was used to estimate the radiation efficiency of a clamped circular plate by Czarnecki, Engel and Panuszka. ${ }^{1}$ The authors also make use of the correlation method for some near field measurements of the sound power of a flat circular plate. ${ }^{2}$ A comprehensive approach to find the radiation efficiency of a spinning annular disk was proposed by Lee and Singh. ${ }^{3}$ A closed path integral technique was used to derive the radiation efficiency of a clamped circular plate by Levine and Leppington. ${ }^{4}$ The technique was applied by Rdzanek to provide some very precise estimates for the sound power of an individual mode and a mode pair valid for the high frequencies. ${ }^{5}$ The technique was later used by Rdzanek and Engel to deal with the magnitudes valid for an annular plate. ${ }^{6,7}$

So far, no theoretical analysis of the complex self power of a guided annular plate has been carried out and no high frequency asymptotics have been presented. Therefore, the aim of this paper is to provide some elementary formulations for the magnitude which would be useful for some highly efficient engineering computations of the total sound power radiated by a guided plate excited in an acoustic fluid.

\section{GOVERNING EQUATIONS OF THE SYSTEM}

A vibrating guided annular plate embedded in a rigid infinite baffle is the source of some time harmonic and axisymmetric acoustic waves radiated into the region above the plate. Low fluid loading and low internal friction are assumed and therefore a linear model of the plate proposed by
Kirchhoff-Love is used. The plate's internal and external radii are denoted as $a$ and $b$, respectively, and $1<s=b / a<\infty$ is the geometric parameter of the plate (see Fig. 1). All the governing equations of a guided annular plate's motion together with their solutions and the directivity pattern were presented in detail in reference ${ }^{8}$. However, it can be useful to quote some of them herein for a better understanding of all the further analysis. The vibrating plate satisfies the following equation of motion in its amplitude form depending on the radial variable $r$ only:

$$
\left(k_{n}^{4} \nabla_{r}^{4}-1\right) W_{n}(r)=0,
$$

where $k_{n}^{2}=\omega_{n} \sqrt{\varrho h / B}$ defines the $n$-th structural wavenumber of the system for the axisymmetric mode $(0, n)$ further referred to as the $n$-th mode for $n=0,1,2,3, \ldots$, where $n=0$ denotes the fundamental elastic vibration mode of the system, i.e. $(0,0), \omega_{n}$ is the corresponding $n$-th eigenfrequency of the system, $\varrho, E$ are the plate's density and Young's modulus, respectively, $D=E h^{3} /\left[12\left(1-v^{2}\right)\right]$ is the plate's bending stiffness, $v$ is its Poisson ratio, and $\nabla_{r}^{2}=\left(d^{2} / d r^{2}\right)+r^{-1}(d / d r)$ defines the $\nabla$ operator for an axisymmetric process.

It was shown in reference ${ }^{8}$ that the corresponding mode shape of the plate satisfying Eq. (1), assumes the form of

$$
W_{n}(r)=A_{n}\left[J_{0}\left(k_{n} r\right)-C_{n} N_{0}\left(k_{n} r\right)\right] .
$$

where $J_{0}, N_{0}$ are the Bessel and Neumann functions of the zero order, respectively, and $A_{n}, C_{n}$ are unknown constats. The boundary conditions of the system are determined by two facts. First, the first order normal radial derivative of the mode shape is equal to zero at both edges of the plate. Second, the external force resisting the transverse deflection of both edges of the plate are also equal to zero for a guided annular plate. Finally, the boundary conditions of the system can be formulated as follows: ${ }^{\mathbf{8} 9}$

$$
\left.\frac{d}{d r} W_{n}(r)\right|_{r=a, b}=0 ;\left.\quad \frac{d}{d r} \nabla_{r}^{2} W_{n}(r)\right|_{r=a, b}=0 .
$$

International Journal of Acoustics and Vibration, Vol. 8, No. 1, 2003 (pp 33-37) 